\title{
Dissipation mechanisms under irreversible deformation
}

\author{
Yury Alyushin ${ }^{1, *}$, and Sergey Gorbatyuk ${ }^{1}$ \\ ${ }^{1}$ National University of Science and Technology "MISiS", 119991, Moscow, Leninsky prospect, 4, RF.
}

\begin{abstract}
The possibility is shown of the transition from reversible to irreversible deformation without additional energy from outside sources by changing the distribution of speeds and accelerations of the particles of the continuum. Two models of irreversible deformation are proposed. The first of which uses a different correlation between transverse and longitudinal deformations at each stage, while the second stage is accompanied by dissipation of energy, stored in the previous elastic stage. For the second model with ideal rigid-plastic continuum, the sequential formation of several families of planes of sliding was substantiated. After the formation of the first one a sliding starts in the second family, which consumes less energy. The consequence is the dissipation and reduction of loadings, the stand sliding on the first family. For the further development of strain it is required to increase loadings up to a critical value, corresponding to the beginning of the first stage. The both models are consistent with the experimentally observed mechanisms of irreversible deformation
\end{abstract}

From among all the many different observed mechanisms of reversible and irreversible deformation slipping and twinning is usually considered as the main [1-3]. Selectivity of sliding plane is due to the line microflaws, called dislocations. Dislocation theory has proved very successful, including for explanation of bending and twisting of sliding planes.

However, by the different theoretic and really observed values of stress for developing dislocations, have moved from simple dislocaton models to complex ones by the early 1980s. The idea of double-level strategy for plasticity description, using autowaves of localized plastic flows, have come up. In work [3] relaxational nature of plastic flow with stress redistribution and reducing their total level through slide processes, was justified. Slide processes activate related spaces with appearance of new pockets thus ensures its autocatalytic nature, when the finish result returns a system to its initial state with subsequent repeating of similar cycles.

Similar mechanisms were found out in monocrystals [4], including a translation slip, when some layers of crystal's atoms slipping over other ones with movements equivalent in size to atom spacing. Deformation mechanisms are the same for any real material [5], but a standard physical analogue is still absent.

There is no a valid mathematical model and on the basis of solids mechanics. Moreover, in modern plasticity theory there is no a standard accounting practice of stress history from an initial to critical state. There are a number of contradictions, for example, about uniform stress and strain states in elementary units and possibility of tangent line ingredient's jump at the surfaces of velocity discontinuity in a perfect plasticity model.

The objective of the work: using general accepted ratios, offer possible models of passing from reversible to irreversible deformation with conversion of stored energy from one species to another with subsequent its dissipation.

From the energy point of view [6-7] irreversible deformations can occur when the volume energy density, increasing under the influence of the elastic deformation, when the critical values can contribute to change the nature of the movement of the particles of the continuum and contributes to the running of the relaxation processes, in which energy is either not changed or reduced, and can return to its original steady value when a significant change in particle size taking into account the existing load.

Specific deformation power $\mathrm{w}$ is equal to the product tensors of the stress $\sigma_{i j}$ and the strain rate $S_{i j}$

$$
\begin{gathered}
w=\sigma_{i j} s_{i j}=\sigma_{x x} x_{t x}+\sigma_{x y} y_{t x}+\sigma_{x z} z_{t x}+ \\
+\sigma_{x y}\left(x_{t y}+y_{t x}\right)+\sigma_{y z}\left(y_{t z}+z_{t y}\right)+\sigma_{z x}\left(z_{t x}+x_{t z}\right)
\end{gathered}
$$

Here $s_{i j}=0,5\left(\partial v_{i} / \partial x_{j}+\partial v_{j} / \partial x_{i}\right), \quad v_{i}=d x_{i} / d t-$ particle movement velocity components. Turning to the spherical tensor $\sigma, s$ and diverter $\sigma_{i j}^{\prime}$ and $s_{i j}^{\prime}$

$$
\sigma_{i j}=\sigma+\sigma_{i j}^{\prime}, \quad S_{i j}=s+s_{i j}^{\prime},
$$

we obtain

$$
\omega=\sigma_{i j} s_{i j}=\sigma \operatorname{div}(\vec{v})+\sigma_{i j}^{\prime} s_{i j}^{\prime} .
$$

Where $\sigma=\left(\sigma_{x x}+\sigma_{y y}+\sigma_{z z}\right) / 3-$ the average stress. The first term describes the power spent on the volume change, the second - in the shape change. Both parts are invariant, the second term can be written in terms of the intensity of tangential stresses $\tau_{e}$ and shear strain rates $S_{i}$,

* Corresponding author: alyushin7@gmail.com 


$$
\begin{gathered}
\omega^{\prime}=\sigma_{i j}^{\prime} s_{i j}^{\prime}=\tau_{e} s_{i}, \\
\tau_{e}^{2}=(1 / 6)\left[\left(\sigma_{x x}-\sigma_{y y}\right)^{2}+\left(\sigma_{y y}-\sigma_{z z}\right)^{2}+\right. \\
\left.+\left(\sigma_{z z}-\sigma_{x x}\right)^{2}+6\left(\sigma_{x y}^{2}+\sigma_{y z}^{2}+\sigma_{z x}^{2}\right)\right], \\
s_{i}^{2}=2\left[\left(s_{x x}-s_{y y}\right)^{2}+\left(s_{y y}-s_{z z}\right)^{2}+\left(s_{z z}-s_{x x}\right)^{2}+\right. \\
\left.+3 / 2\left(s_{x y}^{2}+s_{y z}^{2}+s_{z x}^{2}\right)\right] / 3 .
\end{gathered}
$$

We use the concept of volumetric energy density $u=\delta U / \delta V$ similar to the density of the material $\rho=\delta m / \delta V$. Operator $\delta$ correspond to infinitesimal values of functions, such as volumes, relating to fixed particle with an initial volume of $\delta V_{0}$ at a given moment of time $t>0$. To increment $\delta U$ function of an infinitely small time interval $d t$ we obtain

$$
\begin{gathered}
d \delta U=d(u \delta V)=\left(u_{t} \delta V+u \delta V_{t}\right) d t= \\
=\left(u_{t} R+u R_{t}\right) \delta V_{0} d t=\left[u_{t}+u \operatorname{div}(\vec{v})\right] \delta V d t
\end{gathered}
$$

The right side of the subscript $t$ represents the substantial derivative of the function with respect to time. Comparing equations (1) and (3), and taking into account that the specific power is essentially a volumetric energy density increment rate, it can be argued that the average stress $\sigma$ characterizes the volumetric energy density u (with the dimension of $\mathrm{Pa}$ ), and the specific power to change shape $\omega_{\phi}=\tau_{e} s_{i}$ - the rate of change "volumetric energy density" $u_{t}$ (with the dimension of $\mathrm{Pa} / \mathrm{s}$ ).

In view of this result, the criteria of the limiting value of stress intensity for elastic materials and energy used to change the shape, can be interpreted as the existence of limiting the rate of change (transfer) of energy in solids, as a criterion as the ratio $\tau_{e} / \sigma[8]$ as the existence of the maximum energy the rate of change on each of its particular level (taking into account the denominator).

Based on the above it can be assumed that the failure mechanism in solids is similar to the destruction of heat stroke: very high energy flow can not be transferred to an adjacent volumes and there is a "fusion" of the contact layer with a change in its physical properties.

The amount of plastic deformation processes, workpieces and their constituent particles is not changed. Then the first term on the right side of the equation (1) becomes equal to 0 and the deformation capacity of the product determines the intensities of the stress and strain rate $\omega=\tau_{e} s_{i}$. In accordance with the hypothesis of the constancy of the plastic constant $\tau_{e}=k=$ const can be argued that the deformation capacity mainly determines the intensity of shear strain rate $s_{i}$.

With any addiction $\tau_{e}=\tau_{e}\left(s_{i}\right)$ it can be argued that the development of deformation is possible without additional energy from outside sources by changing the divergence and rotor of vectors of speed $\vec{v}$ and acceleration $\vec{v}_{t}$, as [8]

$$
\begin{gathered}
s_{i}^{2}=2 \operatorname{div}\left(\vec{v}_{t}\right)+ \\
+(\operatorname{rot}(\vec{v}))^{2}-2(\operatorname{div}(\vec{v}))_{t}-(2 / 3)(\operatorname{div}(\vec{v}))^{2} .
\end{gathered}
$$

The equivalence of the above two forms of the equations for $s_{i}^{2}$ (2) and (4) it follows from the relations

$$
f_{t x_{i}}=f_{x_{i} t}+f_{x} x_{t x_{i}}+f_{y} y_{t x_{i}}+f_{z} z_{t x_{i}},
$$

that follow from common rules of differentiation of implicit functions.

Moreover, the transition of reversible to irreversible deformation is possible with a homogeneous velocity field, also due to the localization of deformation in bands slip, when adjacent volumes can be considered rigid.

In particular, in a linear tension the energy is growing during elastic loading through volume and form changes. Volume recover to initial value is only possible with a redundant strain with size reduction across and increase the size in the direction of external force cotemporary. The stored energy shall be not less than critical, which is necessary for launching a mechanism of irreversible deformation. Process must be accompanied by reducing of energy potential of particles and discharge of excess part of energy as a heat.

Consider a possible two-staged mechanism of plastic flow by the example of linear tension in the direction of $x$ axis. At the first stage $0 \leq t \leq t_{s}$ elastic strain occurs $0 \leq \varepsilon_{x} \leq \varepsilon_{s}$ with ratio of transverse to longitudinal deformations $\left|\varepsilon_{y} / \varepsilon_{x}\right|=\mu$ according to a motion equation (time parameter is deformation $\varepsilon_{x}$ )

$$
\begin{gathered}
x=\alpha \exp \left(\varepsilon_{x}\right), \quad y=\beta \exp \left(-\mu \varepsilon_{x}\right), \\
z=\gamma \exp \left(-\mu \varepsilon_{x}\right),
\end{gathered}
$$

where $\alpha=x_{0}, \beta=y_{0}, \gamma=z_{0}$ - are initial coordinates (Lagrange variables). As analysis is expected of both transverse and longitudinal deformations, motion equations were written with logarithmic measure of Hencky deformation $\varepsilon=\ln \left(l / l_{0}\right)$ unlike Cauchy measure $\varepsilon=\left(l-l_{0}\right) / l_{0}$, where $l$ and $l_{0}-$ is a current and initial length. Derivatives $x_{i, p} \equiv \partial x_{i} / \partial \alpha_{p}$ from Euler variables $x_{i} \in(x, y, z)$ by Lagrange variables $\alpha_{p} \in(\alpha, \beta, \gamma)$

$$
\begin{gathered}
\partial x / \partial \alpha \equiv x_{\alpha}=\exp \left(\varepsilon_{x}\right), \\
\partial y / \partial \beta \equiv y_{\beta}=z_{\gamma}=\exp \left(-\mu \varepsilon_{x}\right)
\end{gathered}
$$

determine a relative change of volume $R$, average length of bonds $e$, main energy invariant $\Gamma_{e}^{2}$, and standard deviation $\Gamma$ of bonds' lengths of an infinitesimal parallelepiped from their average value $e$ [9]

$$
R=\delta V / \delta V_{0}=\left|x_{i, p}\right|,
$$




$$
\begin{gathered}
e=\left(e_{\alpha}+e_{\beta}+e_{\gamma}\right) / 3, \\
e_{p}^{2}=x_{p}^{2}+y_{p}^{2}+z_{p}^{2}, \\
\Gamma_{e}^{2}=x_{i, p} x_{i, p}=x_{\alpha}^{2}+x_{\beta}^{2}+x_{\gamma}^{2}+y_{\alpha}^{2}+\ldots .+z_{\gamma}^{2}, \\
\Gamma^{2}=\Gamma_{e}^{2}-3 e^{2} .
\end{gathered}
$$

Stored energy can lead to a spontaneous deformation $\Delta \varepsilon_{x} \geq 0$, which is different from deformation by ratio of transverse to longitudinal deformations at the first stage $\left|\Delta \varepsilon_{y} / \Delta \varepsilon_{x}\right|=\mu^{\prime}$, and at the second stage $t_{s} \leq t \leq t_{k}$ provides volume recover of particles to initial value with a parallel growth of deformation in the direction of tension with increasing standard deviation of bonds' lengths of infinitesimal parallelepiped from their average value

$$
\begin{gathered}
x=\alpha \exp \left(\Delta \varepsilon_{x}\right), \quad y=\beta \exp \left(-\mu^{\prime} \Delta \varepsilon_{x}\right), \\
z=\gamma \exp \left(-\mu^{\prime} \Delta \varepsilon_{x}\right) .
\end{gathered}
$$

To conserve on equations (8) unified with (5) system of time reckoning and therefore deformations, it is enough to use the principle of superposition [6], by replacing of Lagrange variables in equations (5) by the expressions for relevant Euler variables of system (8) if $\varepsilon_{x}=\varepsilon_{s}=$ const

$$
\begin{gathered}
x=\alpha \exp \left(\varepsilon_{s}+\Delta \varepsilon_{x}\right), y=\beta \exp \left(-\mu \varepsilon_{s}-\mu^{\prime} \Delta \varepsilon_{x}\right), \\
z=\gamma \exp \left(-\mu \varepsilon_{s}-\mu^{\prime} \Delta \varepsilon_{x}\right) .
\end{gathered}
$$

At the beginning of the second stage using real values of derivatives (6) for invariant characteristics (7) we get (end values are given for $\mu=0,25$ ) [10]

$$
\begin{gathered}
R=\exp \left[\varepsilon_{s}(1-2 \mu)\right]=\exp \left(\varepsilon_{s} / 2\right)=0,5 \varepsilon_{s}+\varepsilon_{s}^{2} / 8, \\
3 e=3+\varepsilon_{s}(1-2 \mu)+0,5 \varepsilon_{s}^{2}\left(1+2 \mu^{2}\right)= \\
=3+\varepsilon_{s} / 2+9 \varepsilon_{s}{ }^{2} / 16, \\
\Gamma_{e}^{2}=3+2 \varepsilon_{s}(1-2 \mu)+2(1+2 \mu) \varepsilon_{s}{ }^{2}=3+\varepsilon_{s}+3 \varepsilon_{s}{ }^{2}, \\
\Gamma^{2}=x_{\alpha}^{2}+y_{\beta}^{2}+z_{\gamma}^{2}-3 e^{2}=(2 / 3)\left[\varepsilon_{s}(1+\mu)\right]^{2}= \\
=(50 / 48) \varepsilon_{s}^{2} .
\end{gathered}
$$

At the second stage at value $\varepsilon_{x}=\varepsilon_{s}=$ const and growth $\Delta \varepsilon_{x}$ with the derivatives

$$
\begin{gathered}
x_{\alpha}=\exp \left(\varepsilon_{s}+\Delta \varepsilon_{x}\right), \\
y_{\beta}=z_{\gamma}=\exp \left(-\mu \varepsilon_{s}-\mu^{\prime} \Delta \varepsilon_{x}\right),
\end{gathered}
$$

invariant characteristics (7) are

$$
R=\exp \left[\varepsilon_{s}(1-2 \mu)+\Delta \varepsilon_{x}\left(1-2 \mu^{\prime}\right)\right],
$$

$$
\begin{gathered}
3 e=3+\varepsilon_{s}(1-2 \mu)+0,5 \varepsilon_{s}^{2}\left(1+2 \mu^{2}\right)+ \\
+\Delta \varepsilon_{s}\left(1-2 \mu^{\prime}\right)+0,5 \varepsilon_{s}^{2}\left[1+2\left(\mu^{\prime}\right)^{2}\right], \\
\Gamma_{e}^{2}=3+2 \varepsilon_{s}(1-2 \mu)+2 \Delta \varepsilon_{x}\left(1-2 \mu^{\prime}\right)+ \\
+2\left(\varepsilon_{s}+\Delta \varepsilon_{x}\right)^{2}+4\left(\mu \varepsilon_{s}+\mu^{\prime} \Delta \varepsilon_{x}\right)^{2}, \\
\Gamma^{2}=(2 / 3)\left[\varepsilon_{s}(1+\mu)+\Delta \varepsilon_{x}\left(1+\mu^{\prime}\right)\right]^{2}
\end{gathered}
$$

To different combinations of parameter values, appearing in the motion equation and characterizing continuum properties, will be consistent with different reduction value of volumes and quantity of output energy. Volume of particle recovers its initial value if the additional deformation satisfies the condition

$$
\Delta \varepsilon_{x}=-\varepsilon_{s}(1-2 \mu) /\left(1-2 \mu^{\prime}\right) .
$$

In order that it remains positive (in this case the tension of specimen is continuing) shall be met condition $\mu^{\prime}>0,5$. Of course, a complete reduction of volume is not necessary and a range of possible change $\mu^{\prime}$ may be too broad at the second stage. Reduction of volume also depends on a deformation ratio $\Delta \varepsilon_{x} / \varepsilon_{s}$. A way of full reduction of volume is possible, for example, if deformations remained equal at the first and second stages $\varepsilon_{s}=\Delta \varepsilon_{x}, \quad$ then $\mu^{\prime}=1-\mu, \quad e_{\alpha}=\exp \left(2 \varepsilon_{s}\right)$, $e_{\beta}=e_{\gamma}=\exp \left(-\varepsilon_{s}\right)$. From (12) we get

$$
R=1, \quad e=1+\varepsilon_{s}^{2}, \Gamma_{e}^{2}=3+12 \varepsilon_{s}^{2}, \Gamma^{2}=6 \varepsilon_{s}^{2} .
$$

As a result the volume recovers its initial value with significant changes of particle sizes, deviation $\Gamma^{2}$ increases almost in 6 times.

Using ratios $\tau_{p i}=\varphi x_{i, p}$ between stresses $\tau_{p i}$ and deformations of Lagrange $x_{i, p}$ [6], at the end of the first stage the total relative energy gain of particle is

$$
E_{\text {деp }}=\varphi \varepsilon_{s}\left(1+3 \varepsilon_{s}\right) .
$$

According to equations (9) relieving at the second stage, when the volume recovers its initial value, reduces energy density of particles till $E_{\phi}=6 \varphi \varepsilon_{s}{ }^{2}$, work of external forces almost completely transformed in heat and goes into environment [7-9]

$$
E_{\text {дисс }}=\varphi \varepsilon_{s}\left(1-3 \varepsilon_{s}\right) .
$$

Given that the second stage must be accompanied by a release of heat then at this stage (isotropic continuum) the deformation ratio will be $\mu^{\prime}=1$, because deformations from increasing of temperature are the same in all directions $\Delta \varepsilon_{y}=\Delta \varepsilon_{x}$. In this case the full reduction of initial volume is possible if $\Delta \varepsilon_{x}=0,5 \varepsilon_{s}$ with dissipation of stored energy. 
The described mechanism includes the changes of deflection behavior at the second stage, even allowing homogenous deformation by analogy with elastic area. This complicates understanding of dissipation mechanism, which is usually identified with Lueder's bands [1-4]. As point heat sources, such slidings stimulate dissipation of stored energy and periodic process execution of plastic flow in surrounding volumes.

To build the simplest energy model of localization of deformation zones, can use properties of an ideal plasticrigid continuum and extremum principle [6], under which the power, required for deformation whatever kinematically-admissible field of velocities, is always higher than in a real process of deformation. It allows to find an upper bound of power or deformation loading and field of velocities under conditions close to actual practice.

For the plastic-rigid continuum kinematicallyadmissible is a field of velocities which met the condition of volume stability and boundary conditions for velocities [11]. The feature of the ideal plastic-rigid continuum is that a material may be both in solid and plastic states by intensity of tangential stress $\tau_{e}=k$. Under deformation of material with such properties inside object, can appear slide surfaces (fig. 1,2), on both sides of which material is hard. These surfaces are similar to contacting surfaces of two, moving relative to each other, hard objects, but influencing tangential stress on them shall comply the passage condition of material plastic state and $\tau_{\max }=k$.

For example, under tension of rectangular-sectioned specimen $h \times b$ suggests that sliding occurs on plane $A B C D$ (fig. 1) with angles $\alpha$ and $\beta$. From condition of vector's complanarity $A B, A C$ and $A D$ [12] find an equation of plane of sliding $A B C D$

$$
x \operatorname{tg} \alpha+y \operatorname{tg} \beta-z=0
$$

and its area if cross-section $f_{0}=b h$

$$
f_{12}=b h\left(1+\operatorname{tg}^{2} \alpha+\operatorname{tg}^{2} \beta\right)^{1 / 2} .
$$

The volume of specimen remains immobile below the plane $A B C D$. In order to comply with boundary condition a displacement velocity of upper part relatively lower one $\vec{v}_{12}=u \vec{i}+v \vec{j}+w \vec{k}$ shall be parallel to the plane of sliding with ratio of components

$$
w=v_{0}=u \operatorname{tg} \alpha+v \operatorname{tg} \beta
$$

and velocity of point's relative movement on both sides of plane $A B C D$

$$
v_{12}=\sqrt{u^{2}+v^{2}+(u \operatorname{tg} \alpha+v \operatorname{tg} \beta)^{2}} .
$$

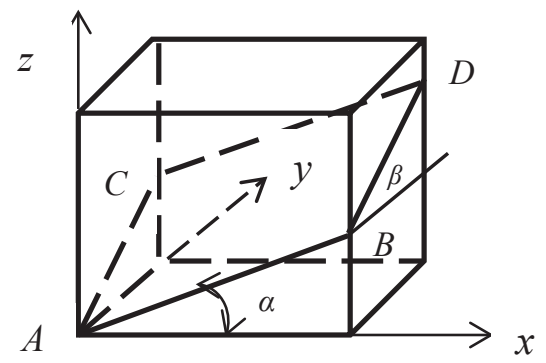

Fig. 1. Plane of sliding under linear tension.

Under the tangential stresses $\tau_{\max }=k$ the capacity $W_{12}=k f_{12} v_{12}$ is required on shear, which should be from external reference source. Under specific loading on upper end $q$ from condition of capacities' equality of external and internal forces

$$
q b h v_{0}=k f_{12} v_{12}
$$

find

$$
\frac{q}{k}=\frac{\sqrt{1+\operatorname{tg}^{2} \alpha+\operatorname{tg}^{2} \beta}}{\operatorname{tg} \alpha+\psi \operatorname{tg} \beta} \sqrt{1+\psi^{2}+(\operatorname{tg} \alpha+\psi \operatorname{tg} \beta)^{2}},
$$

where value $\psi=v / u$ gives the ratio of displacement velocities of the upper part in $x$ and $y$ direction relatively.

For all values $\psi, \alpha, \beta$ velocity field is kinematically-admissible. As these characteristics are independent, their values shall be defined from minimum condition of specific loadings.

$$
\partial q / \partial \alpha=0, \quad \partial q / \partial \beta=0, \quad \partial q / \partial \psi=0 .
$$

Subject to the conditions

$$
\psi \operatorname{tg} \alpha=\operatorname{tg} \beta ; \quad \operatorname{tg}^{2} \alpha+\operatorname{tg}^{2} \beta=1,
$$

the specific loadings (13) are $q=2 k$, including for the state of plane strain if $\alpha=45^{\circ}$ and $\beta=0, \psi=0$. If properties are similar (isotropic continuum) in $x$ and $y$ directions then can be taken $\psi=1$ for comparable sizes $b \approx h$ and from the second equation get $\alpha=\beta=$ $35^{\circ} 16^{\prime} 20^{\prime \prime}$. Exactly this result has been confirmed experimentally [9].

Loading diagram changes after the appearance of plane of sliding. Now the lower part is under tangential stress $\tau_{\text {max }}=k$ on plane $A B C D$, which for simplicity of graphical representation will be characterized by a slope of its normal relative to vertical (a tension load) by angle $\alpha_{1}$. While not excluding the appearance of new plane of sliding with angle $\alpha_{2}$. In fig.2 physical plane and velocity hodograph shows a velocity of mobile zone 2 between 0 and 1 zones under conditions of equal of normal components of velocity on all planes of sliding. On plane $f_{12}$ between 1 and 2 zones tangential stresses $\tau_{12}=\tau_{\text {max }}=k$ generate a force $F_{12}=k b h / \cos \alpha_{1}$, 
directed at angle $\alpha_{1}$ to horizontal. The power is defined by scalar product of vectors of force and velocity with angle between them $\left(\alpha_{1}-\alpha_{2}\right)$

$$
W_{12}=k v_{02}\left(b / \cos \alpha_{1}\right) \cos \left(\alpha_{1}-\alpha_{2}\right) \text {. }
$$

At the surface of new plane power consumption is

$$
W_{02}=k v_{02}\left(b / \cos \alpha_{2}\right) \text {. }
$$

Deformation with the formation of secondary system of sliding is possible if $W_{12} \geq W_{02}$ or

$$
\tau_{12} v_{02}\left(b / \cos \alpha_{1}\right) \cos \left(\alpha_{1}-\alpha_{2}\right) \geq \tau_{02} v_{02}\left(b / \cos \alpha_{2}\right)
$$

If $\tau_{12}=\tau_{02}=k$ all angle values $0 \leq \alpha_{2} \leq \alpha_{1}$ meet this condition, put in other words, the secondary bands shall be to initial ones from parallel to horizontal.

The most probable will be ones for which the required power are minimum. The location of such planes specifies a condition for minimum ratio

$$
\theta=\frac{\cos \left(\alpha_{1}-\alpha_{2}\right) \cos \alpha_{2}}{\cos \alpha_{1}}
$$

From $d \theta / d \alpha_{2}=0$ get

$$
\sin \left(\alpha_{1}-\alpha_{2}\right) \cos \alpha_{2}-\cos \left(\alpha_{1}-\alpha_{2}\right) \sin \alpha_{2}=0
$$

where $\sin \left(\alpha_{1}-2 \alpha_{2}\right)=0$ or $\alpha_{2}=\alpha_{1} / 2$.

Because the analyze made for arbitrary values of angles $\alpha_{1}$ and $\alpha_{2}$, this result may be expanded to any follow-up surfaces of slide.

Estimates suggest that sliding on planes of follow-up systems is possible, in this case at the previous family shear capacity exceeds the required up to $17,2 \%$ for the second system of sliding (with tilting angle $22,5^{\circ}$ ), just for $4 \%$ for the third one(with tilting angle $11,25^{\circ}$ ) and just for $1 \%$ for the forth one (with tilting angle $5,625^{\circ}$ ).

Suchwise, every previous system of sliding may create the follow-up one, but their concurrent existence is impossible. Applied in zone 1 specific loading $q=2 k$ is not enough to maintain sliding at the first two families of deformation bands. With using sliding velocities $v_{02}$ and $v_{12}$ on surfaces $f_{02}$ and $f_{12}$ with tilting angles $\alpha_{2}$ and $\alpha_{1}$ these specific loadings produce the power

$$
W_{q}=q b h\left(v_{02} \sin \alpha_{2}+v_{12} \sin \alpha_{1}\right) \text {, }
$$

while consumable power (if shear stress $k$ ) is

$$
W_{k}=W_{02}+W_{12}=k b h\left(v_{02} / \cos \alpha_{2}+v_{12} / \cos \alpha_{1}\right) \text {. }
$$

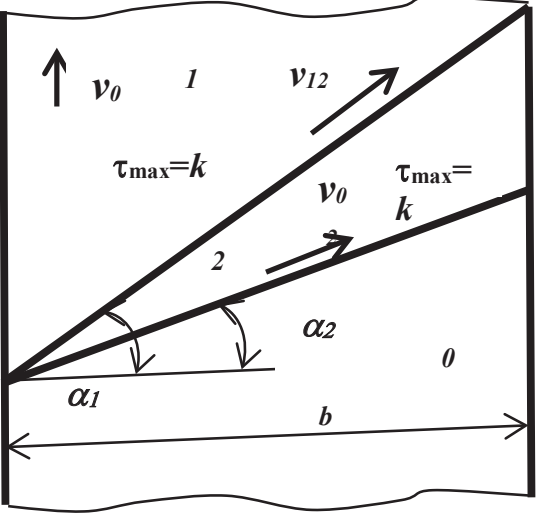

a)

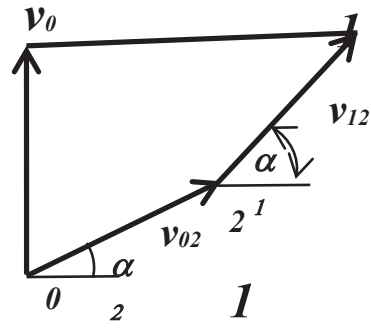

b)

Fig.2. Scheme of pre- and post- deformation bands: a) physical plane b) velocity hodograph.

Indicate velocity ratio $\psi=v_{02} / v_{12}$, and then a condition of capacity equality (15) and (16) can record as

$$
q v_{12}\left(\psi \sin \alpha_{2}+\sin \alpha_{1}\right)=k v_{12}\left(\psi / \cos \alpha_{2}+1 / \cos \alpha_{1}\right) \text {. }
$$

If $q=2 k$ and $\alpha_{1}=45^{\circ}$ the equality for the first family

$$
2\left(\psi \sin \alpha_{2}+\sin \alpha_{1}\right)=\psi / \cos \alpha_{2}+1 / \cos \alpha_{1}
$$

is only met if $\psi=0$ or $\alpha_{2}=\alpha_{1}$.

Suchwise, if achieved the specific loadings $q=2 k$ a primary system of sliding appears with tilting angle $\alpha_{1}=45^{\circ}$. Below this plane in the volume of specimen shear on plane with tilting angle $\alpha_{2}=22,5^{\circ}$ becomes the preferred, because in the arisen conditions such deformation demands less power. It is not excluded the appearance of post- deformation bands, right up to horizontal ones. But external forces cannot support coexistence of two families, that's why the sliding comes to an end on surface between 0 and 1 zones.

On the secondary sliding plane power reduction of deformation leads to load relaxation at $17,2 \%$ and appearance of "yield drop" on diagram "force-strain" which is observed experimentally [14]. Under static tension in condition of plane strain distraction of plated specimens under tension more often occurs at an angle not $45^{\circ}$, but about $21^{0}$ [13]. Consequently, slides are more significant in the second family than in the first one.

The considered model of the transition of reversible to irreversible deformations agree with the experimental data. 


\section{References}

1. R. Honecomb, Plastic deformation of metals (M.: Mir, 1972)

2. S.M. Gorbatyuk, A.V. Kochanov, Method and equipment for mechanically strengthening the surface of rolling-mill rolls, Metallurgist, 56 (3-4), pp. 279-283 (2012)

3. L. B. Zuev, V. I. Danilov, S. A. Barannikova, Physics of plastic flow microlocalization (Novosibirsk: Nauka, 2008)

4. S.M. Gorbatyuk, V.M. Pavlov, A.N. Shapoval, M.S. Gorbatyuk, Experimental use of rotary rolling mills to deform compacts of refractory metals, Metallurgist, 42 (5-6), pp. 178-183 (1998)

5. E. Nikolaeva, Shear mechanisms of plastic deformation of single crystals (Perm: Publishing house Perm. GOS. tehn. University press, 2011)

6. Yu. Alyushin, Energy principles of mechanics (Lambert Academic Publishing, 2016)

7. Yu. Alyushin, Energy model of reversible and irreversible deformations in the space of Lagrangian variables, Advanced technology plastic deformation, M.: NITU MISIS, pp. 44-67 (2009)

8. Yu. Alyushin, M. Skripalenko, Energy peculiarities and accelerations during reversible and irreversible deformations, Journal of Machinery Manufacture and Reliability, 40 (2), pp. 154-160 (2011).

9. Yu. Alyushin, The generalized model of reversible and irreversible deformation describing the processes in Lagrange form, «KuznechnoShtampovochnoe Proizvodstvo», 6, pp. 2-5 (1997).

10. Yu. Alyushin, Mechanics of deformation processes in the space of Lagrangian variables (M.: Mechanical Engineering, 1997)

11. A. Tomlenov, Mechanics of metal forming (M.: Mashgiz, 1963)

12. Yu. Alyushin, Theory of metal forming (Rostov-onDon: RISHM, 1977)

13. A. Nadai Flow and fracture of solids (M.: Mir, 1969)

14. D. Bell Experimental foundations of mechanics of deformable solids (M.: Nauka, 1984) 